\title{
A type-2 fuzzy community detection model in large-scale social networks considering two-layer graphs
}

\author{
Mansoureh Naderipour ${ }^{\mathrm{a}}$, Mohammad Hossein Fazel Zarandi ${ }^{\mathrm{a}, *}{\underline{\text { Susan } \text { Bastani }^{\mathrm{b}}}}^{\mathrm{b}}$
}

${ }^{a}$ Department of Industrial Engineering, Amirkabir University of Technology (Polytechnic of Tehran), P.O. Box 15875-4413, Tehran, Iran.

${ }^{\mathrm{b}}$ Department of Social Sciences and Economics, Alzahra University, 19938-93973 Tehran, Iran.

\begin{abstract}
This paper mainly aims to identify communities with different interactions between nodes in complex networks. Community detection algorithms partition vertices into denselyconnected components in a complex network. In recent researches, a node is related to multiple aspects of relationships resulting in new challenges in social networks. The two aspects of relationships could be shown as a two-layer graph which comprises two graphs dependent on each other; and each graph shows a specific aspect of the interaction. In this research, a new community detection model is proposed based on the possibilistic cmeans clustering model considering two-layer graphs (PCMTL) in order to detect overlapping communities based on the two-layer graphs using both structural and attribute similarities in large-scale social networks. The nodes are assigned to communities by upper and lower membership values that are indicative of the degree of belonging to the communities through type-2 fuzzy membership values, and the suggested values of interval type-2 fuzzy membership determine how a node belongs to a community with regard to two different aspects of interactions in a two-layer graph. Moreover, according to the proposed model, a validity index is introduced to assess the suggested model in comparison to the approach existing in the literature. Ultimately, two artificial and two real large-scale social networks are used to validate the performance of the suggested model.
\end{abstract}

Keywords: Community detection, Overlapping communities, Structural/attribute similarities, Two-layer graph, Type-2 fuzzy clustering. 